\title{
A robust two-level incomplete factorization for (Navier-)Stokes saddle point matrices
}

\author{
Fred W. Wubs* and Jonas Thies ${ }^{\dagger}$
}

October 17, 2018

\begin{abstract}
We present a new hybrid direct/iterative approach to the solution of a special class of saddle point matrices arising from the discretization of the steady incompressible Navier-Stokes equations on an Arakawa C-grid. The two-level method introduced here has the following properties: (i) it is very robust, even close to the point where the solution becomes unstable; (ii) a single parameter controls fill and convergence, making the method straightforward to use; (iii) the convergence rate is independent of the number of unknowns; (iv) it can be implemented on distributed memory machines in a natural way; (v) the matrix on the second level has the same structure and numerical properties as the original problem, so the method can be applied recursively; (vi) the iteration takes place in the divergencefree space, so the method qualifies as a 'constraint preconditioner'; (vii) the approach can also be applied to Poisson problems.

This work is also relevant for problems in which similar saddle point matrices occur, for instance when simulating electrical networks, where one has to satisfy Kirchhoff's conservation law for currents.

Keywords: Saddle point problem, indefinite matrix, $\mathcal{F}$-matrix, incomplete factorization, grid-independent convergence, Arakawa C-grid, incompressible (Navier-)Stokes equations, constraint preconditioning, electrical networks.
\end{abstract}

\section{Introduction}

Presently, a typical computational fluid dynamics (CFD) problem may involve millions of unknowns. They represent velocities and pressures on a grid and are determined by solving a large sparse linear system of equations. Robust numerical methods are needed to achieve high fidelity. Therefore one often resorts to direct (sparse) solvers. In general such a method does not fail as long

\footnotetext{
*Johan Bernoulli Institute of Mathematics and Computing Science, University of Groningen, P.O.Box 407, 9700 AK Groningen, The Netherlands, Email: f.w.wubs@rug.nl

†j.thies@rug.nl
} 
as the used precision is enough to handle the posedness of the problem. However, there are two disadvantages to direct methods. Firstly, the amount of memory required for the factorization is not linear in the number of unknowns, and when increasing the problem size one may encounter memory limitations sooner than expected due to fill generated in the factors. Secondly, all the new elements in the factorization have to be computed, so that the computing time grows sharply, too. This holds especially for 3D problems, where the computational complexity of direct methods for partial differential equations (PDEs) grows with the square of the number of unknowns.

For this reason one has to resort to iterative methods for very large applications. Such methods perform a finite number of iterations to yield an approximate solution. In theory the accuracy achieved increases with the number of iterations performed. However, iterative methods are often not robust for complex problems. The iteration process may stall or diverge and the final approximation may be inaccurate. Furthermore they often require custom numerics such as preconditioning techniques to be efficient.

The hybrid direct/iterative approach presented here seeks to combine the robustness of direct solvers with the memory and computational efficiency of iterative methods. It is based on the direct method recently developed for the Stokes $\mathcal{F}$-matrix by De Niet \& Wubs (2009), which has the property that the fill does not increase in the "gradient" and "divergence" part of the matrix. To extend this to an incomplete factorization preconditioner one only has to drop velocityvelocity couplings to limit the amount of fill. We perform a non-overlapping domain decomposition of the grid, and eliminate the interior velocities using a direct method. For the remaining variables a Schur-complement problem has to be solved, which we do by a Krylov subspace method preconditioned by a novel incomplete factorization preconditioner.

In this paper we start out by giving a survey of previous research in section 2 , In section 3 we will describe the problem in more detail and review the direct method developed by De Niet \& Wubs (2009). In section 4 we will introduce the proposed iterative procedure based on this direct method. In section 5 we present numerical results for a series of increasingly complex CFD problems: the Poisson, Darcy, Stokes and Navier-Stokes equations.

We conclude in section 6 by summarizing the method and results and giving an outlook on future work.

\section{Survey of previous work}

By Benzi et al. (2005) a survey is given of methods currently in use to solve linear systems from fluid flow problems. In many cases saddle point problems can be solved efficiently by a Krylov subspace iteration (Van der Vorst 2003) combined with appropriate preconditioning (Benzi \& Olshanskii 2006, Benzi et al. 2005, De Niet \& Wubs 2007, Elman et al. 2002, Kay et al. 2002, Elman et al. 2008). Often a segregated approach is used, i.e. the velocities are solved independently from the pressures. This results in inner and outer iterations, 
the former for the independent systems, and the latter to bring the solutions of these systems into balance with each other. We advocate a fully coupled approach.

The idea of combining direct and iterative methods has been used by Hénon \& Saad (2006) and Gaidamour (2008) to solve general sparse linear systems arising from the discretization of scalar PDEs. As in this paper, they reduce the problem to a Schur-complement system on the separators of a domain decomposition. The Schur-complement system is solved iteratively using an ILU factorization. As the structural and numerical properties are not explicitly preserved, robustness and grid-independence cannot be ascertained for indefinite problems.

Recently, De Niet \& Wubs (2009) proposed a direct method for the solution of $\mathcal{F}$-matrices, of which the incompressible Stokes equations on an Arakawa Cgrid are a special case. This special purpose method reduces fill and computation time while preserving the structure of the equations during the elimination. It still suffers from the weaknesses of direct methods, but only the number of velocity-velocity couplings increases, not the number of velocity-pressure couplings. We believe that a better understanding of the $\mathcal{F}$-matrices will lead to generalizations that are of interest to a broader class of indefinite problems and note that there are applications outside the field of fluid mechanics, e.g. in electronic circuit simulations (Vavasis 1994), which lead to $\mathcal{F}$-matrices.

For incompressible flow one has to satisfy an incompressibility constraint: the velocity should be divergence-free. We remark that our iterative technique does not violate the divergence constraint and therefore belongs to the class of 'constraint preconditioners' (Keller et al. 2000). For details see section 4.5 .

\section{$3 \quad \mathcal{F}$-matrices and the direct solution method}

In this paper we study the solution of the equation

$$
K x=b,
$$

where $K \in R^{(n+m) \times(n+m)}(n \geq m)$ is a saddle point matrix that has the form

$$
K=\left[\begin{array}{ll}
A & B \\
B^{T} & 0
\end{array}\right],
$$

with $A \in R^{n \times n}, B \in R^{n \times m}$. Special attention is given to a class of saddle point matrices known as $\mathcal{F}$-matrices. We start out by defining the gradient matrix in which the $\mathcal{F}$-matrix is expressed.

Definition 1 A gradient-matrix has at most two nonzero entries per row and its row sum is zero.

We have chosen the name gradient-matrix, because this type of matrix typically results from the discretization of a pressure gradient in flow equations. It is important to note that the definition allows a gradient-matrix to be non-square. Now we can define the $\mathcal{F}$-matrix. 


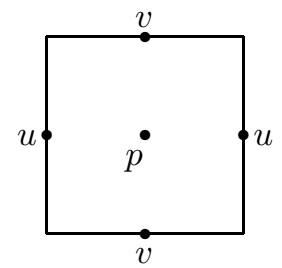

Figure 1: Positioning of velocity $(u, v)$ and pressure $(p)$ variables in the C-grid.

Definition 2 A saddle point matrix (2) is called an $\mathcal{F}$-matrix if $A$ is positive definite and $B$ is a gradient-matrix.

The definition is due to Ti̊ma (2002). $\mathcal{F}$-matrices occur in various fluid flow problems where Arakawa A-grids (collocated) or C-grids (staggered, see figure1) are used. For example, in Arioli \& Manzini (2003) the discretization of Darcy's equation in ground-water flow results in an $\mathcal{F}$-matrix. They also occur in electronic network simulations (Vavasis 1994).

\subsection{The algorithm for the direct approach}

Many of the standard algorithms have in common that they compute a fillreducing ordering for $K$ and then somehow adapt it to make it feasible: a factorization is feasible if it does not break down due to a zero pivot. The delay of elimination (through pivoting) will give an increase in computing time and may lead to increased fill in the factors. To preclude this inefficiency we propose a different approach. Suppose the sets of all velocities and pressures are denoted by $V$ and $P$, respectively. The respective elements will be called $V$-nodes and $P$-nodes. The idea is to first compute an ordering for the $V$-nodes based on a graph that contains information of the whole matrix, and then insert the $P$-nodes appropriately. Assume that we have an elimination ordering on $V$, then we use the following simple rule to insert $P$-nodes into the ordering:

Rule 1 during Gaussian elimination with $K$, whenever a $V$-node is to be eliminated which is connected to a $P$-node, these nodes are eliminated together using a $2 \times 2$ pivot.

With this rule we get as many $2 \times 2$ pivots as there are $P$-nodes. Only if due to elimination a $V$-node becomes totally disconnected from $P$ it can be eliminated on its own.

As all $P$-nodes are eliminated together with a $V$-node in pivots of the form

$$
\left(\begin{array}{ll}
\alpha & \beta \\
\beta & 0
\end{array}\right),
$$


the factorization is always feasible and additional pivoting is not required.

If we apply this rule to an ordering on $V$ that is constructed as a fill-reducing ordering for $A$, the resulting ordering for $K$ will not be fill-reducing in general. To ensure that the final ordering is fill-reducing we have to use information about the whole matrix, i.e. the fill patterns of $B$ and $B^{T}$ have to be taken into account. This is the case if the ordering for $V$ is fill-reducing for the fill pattern $F(A) \cup F\left(B B^{T}\right)$, where $F(A)$ denotes the fill pattern of $A$. This graph is an envelope for the fill that will be created by elimination of the nodes in $P$. In many cases this will be equal to $F\left(A+B B^{T}\right)$, but to avoid possible cancellation in the addition we will use the matrix $F(A) \cup F\left(B B^{T}\right)$. Summarizing we get the following algorithm:

Algorithm 1 To compute a feasible fill-reducing ordering for the saddle point matrix $K$ :

1. Compute a fill-reducing ordering for the $V$-nodes based on $F(A) \cup F\left(B B^{T}\right)$.

2. Insert the P-nodes into the ordering according to rule 1 .

The $P$-nodes (step 2) can be inserted dynamically during Gaussian elimination, which means that we have to adapt the elimination process. The elimination is performed using the fill-reducing ordering on $V$ and applying rule 1. This also takes into account that $V$-nodes initially coupled to $P$-nodes become decoupled because of cancellation, which is a rather common phenomenon (see section 4.2). This is different from just combining pressures with velocities beforehand (static pivoting).

The above method has structure preserving properties which we list in the theorems below. The first two are taken from De Niet \& Wubs (2009), where they were proved for symmetric positive definite $A$. Along the same lines they can be proved for non-symmetric positive definite $A$.

Theorem 1 If $K$ is an $\mathcal{F}$-matrix, all Schur complements $K^{(l)}$ are $\mathcal{F}$-matrices.

This means that the $A$ part will remain positive definite and the $B$ part will have at most 2 entries per row in any step of the elimination. The latter allows us to keep the $B$ part exact during the incomplete factorization.

Theorem 2 The $B$ part in all Schur complements is independent of the size of the entries in the $A$ part.

Theorem 3 If initially $B$ has entries with magnitude one, then this will remain so during the elimination.

Theorem 4 If a $P$-node is not eliminated together with the first $V$-node it is attached to, the next Schur complement will not be an $\mathcal{F}$-matrix.

Proof Consider the matrix in Equation 3 in the next section. It is clear that using only $\alpha$ as pivot will give a contribution in the zero block.

Results of the direct method were shown with AMD (Amestoy et al. 1996) as fill reducing ordering in De Niet \& Wubs (2009). 


\section{Structure preserving incomplete factorization}

In this section we want to develop an incomplete factorization based on the direct method described so far. First we will introduce the domain decomposition we use and then we will illustrate that simply applying a dropping strategy to the $A$ part may not give the desired result when there are couplings to $P$-nodes. We then proceed to develop a combination of orthogonal transformations and dropping that leads to grid-independent convergence, limits fill-in and keeps the divergence constraint intact.

Assumption. For this section we will assume that the entries in $B$ have equal magnitude. This is not a restriction because it can be achieved by scaling the rows of an arbitrary gradient matrix $B$. If $D B$ gives the desired matrix, our new matrix will be

$$
\left[\begin{array}{cc}
D A D & D B \\
B^{T} D & O
\end{array}\right]
$$

Observe that the post-scaling means that the $V$-nodes will be scaled. For NavierStokes on a stretched grid (see section 5.4) the scaling is such that we get as new unknowns the fluxes through the control cell boundaries.

\subsection{Domain decomposition}

The first step of the proposed method is to construct a non-overlapping decomposition of the physical domain into a number of subdomains. This can be done by applying a graph-partitioning method like Metis (Karypis \& Kumar 1998) or similar libraries to $F(A) \cup F\left(B B^{T}\right)$. Metis has been tested successfully, but for this paper we use a manual partitioning into equally-sized square subdomains. (For the Navier-Stokes equations we used a stretched grid, so in that case they are not square and equally-sized in physical space but in the number of unknowns).

Then we introduce a minimal overlap: two adjacent subdomains share one layer of velocity nodes, whereas pressure nodes are not shared among subdomains. Variables belonging to exactly one subdomain are said to be interior variables. Velocities connecting to interior variables in more than one subdomain form separators of the subdomains they connect to. The separator velocities are complemented by an arbitrary single $P$-node per subdomain. When eliminating the interior variables in the next step, this ensures that the subdomain matrix is non-singular (in physical terms the pressure level inside the subdomain is fixed). We remark that

(i) the domain decomposition can be seen as a Nested Dissection ordering as may be used in step 1 of Algorithm 1 stopped at a certain subdomain size (see also (Toselli \& Widlund 2005) in the paragraph "Schur Complement Systems" starting on page 262); 
(ii) we used horizontal and vertical separators as depicted for two domains in fig. 2 A better choice may be to use skew separators $\left( \pm 45^{\circ}\right)$, leading to about half the $V$ nodes on the separator for subdomains of similar size. Both approaches yield the same number of $V$ nodes with couplings to $P$ nodes in the Schur-complement, and we chose for ease of programming here;

(iii) we use the decomposition primarily for numerical reasons and the number of subdomains will typically be much larger than the number of processors in a parallel computation.

We can now eliminate the interior variables, leading to a Schur-complement problem for the separator velocities and remaining pressures. The remainder of this section is devoted to constructing an incomplete factorization preconditioner for this Schur-complement, so that it can be solved efficiently by a Krylov subspace method.

\subsection{The dropping problem}

Consider the following matrix, which occurs in any elimination step with a $2 \times 2$ pivot:

$$
\left[\begin{array}{cc|cc}
\alpha & \beta & a^{T} & b^{T} \\
\beta & 0 & \hat{b}^{T} & 0 \\
\hline a & \hat{b} & \hat{A} & \hat{B} \\
b & 0 & \hat{B}^{T} & O
\end{array}\right] .
$$

When performing the elimination step, a multiple of $\hat{b} \hat{b}^{T}$ is added to $\hat{A}$. This does not introduce new fill if $\hat{A}$ is dense. But if we replaced $\hat{A}$ by a sparse matrix by dropping, the matrix would be filled again as $\hat{b}$ is typically dense.

This is a common phenomenon. Consider, for example, the two-domain case in fig. 2. After eliminating the interior variables, many of the $V$-nodes on the separator are coupled to the two remaining $P$-nodes. Assume that we drop all connections between the $V$-nodes on the separator, so in the above matrix (3), $\hat{A}$ is replaced by its diagonal, and $a$ becomes zero; $\hat{b}$ is a dense vector, $\hat{B}$ has an associated dense column with opposite sign, and $b^{T}$ has a nonzero at the same column position with sign opposite to that of $\beta$. When eliminating one " $V$-node $P$-node" pair, all the $V$-nodes on the separator become detached from $P$ and $\hat{A}$ becomes dense.

From the above we learn that we should try to get more zeros into $\hat{b}$. Or stated otherwise, we should try to decouple the $V$-nodes on the separator from the $P$-nodes as far as possible.

\subsection{Orthogonal operators to decouple $V$ - and $P$-nodes}

One idea to get rid of unwanted pressure couplings is to simply drop them. However, the fill in the $B$-part is already modest and an exact $B$-part is attractive, 


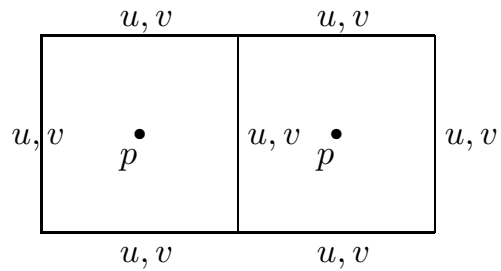

Figure 2: Velocity separators $(u, v)$ and pressure per domain $(p)$ in a 2-domains case.

as discussed in section 4.5. Fortunately we can do better. Consider the square domain decomposition (fig. 2), extended periodically so that every subdomain is bounded by four separators from the neighboring subdomains. The Schurcomplement for the separator velocities and remaining pressures has about the following form (the $V$-nodes in the corners are neglected here, in practice they form 'separators of the separators' and get a block of their own):

$$
\left[\begin{array}{cccccc}
A_{11} & B_{1} & A_{12} & A_{13} & O & O \\
B_{1}^{T} & O & B_{21}^{T} & B_{31}^{T} & O & O \\
A_{21} & B_{21} & A_{22} & O & A_{24} & B_{22} \\
A_{31} & B_{31} & O & A_{33} & A_{34} & B_{32} \\
O & O & A_{42} & A_{43} & A_{44} & B_{42} \\
O & O & B_{22}^{T} & B_{32}^{T} & B_{42}^{T} & O
\end{array}\right]\left[\begin{array}{l}
v_{1} \\
p_{1} \\
v_{2} \\
v_{3} \\
v_{4} \\
p_{2}
\end{array}\right]=\left[\begin{array}{l}
b_{v_{1}} \\
b_{p_{1}} \\
b_{v_{2}} \\
b_{v_{3}} \\
b_{v_{4}} \\
b_{p_{2}}
\end{array}\right]
$$

Here $v_{1}$ contains the $V$-nodes on a certain separator, $p_{1}$ contains the two $P$ nodes from the adjacent subdomains; $v_{2}$ and $v_{3}$ contain the $V$-nodes from other separators around these subdomains, respectively. $v_{4}$ and $p_{2}$ represent the remaining $V$ - and $P$-nodes in the Schur-complement (separator velocities and pressures not connected to the separator under consideration).

Now $B_{1}$ only contains two dense columns, equal up to a sign. So by using an orthogonal transformation $H$, e.g. a Householder reflection, we can transform $B_{1}$ into a matrix with only entries on a certain row, usually the first. Applying $H$ to the first block row and column from left and right, respectively, we obtain the following system (note that the properties of the matrix are preserved by the orthogonal transformation):

$$
\left[\begin{array}{cccccc}
H^{T} A_{11} H & H^{T} B_{1} & H^{T} A_{12} & H^{T} A_{13} & O & O \\
\left(H^{T} B_{1}\right)^{T} & O & B_{21}^{T} & B_{31}^{T} & O & O \\
A_{21} H & B_{21} & A_{22} & O & A_{24} & B_{22} \\
A_{31} H & B_{31} & O & A_{33} & A_{34} & B_{32} \\
O & O & A_{42} & A_{43} & A_{44} & B_{42} \\
O & O & B_{22}^{T} & B_{32}^{T} & B_{42}^{T} & O
\end{array}\right]\left[\begin{array}{c}
H^{T} v_{1} \\
p_{1} \\
v_{2} \\
v_{3} \\
v_{4} \\
p_{2}
\end{array}\right]=\left[\begin{array}{c}
H^{T} b_{v_{1}} \\
b_{p_{1}} \\
b_{v_{2}} \\
b_{v_{3}} \\
b_{v_{4}} \\
b_{p_{2}}
\end{array}\right] .
$$

The Householder matrix is a full matrix (though its application is cheap if its defining form is exploited) and would destroy the sparsity. However, the 
matrices $A_{11}, A_{12}$ and $A_{13}$ are typically already dense (see remark below), so not much is lost and we have gained a lot: we decoupled all but one of the $V$ nodes on the separator from the $P$-nodes. The decoupled ones can be eliminated on their own now.

Remark 1 The fill of $A_{11}, A_{12}$ and $A_{13}$ depends on the problem at hand. For the 2D Stokes-equations in the absence of the pressure terms we get two decoupled Poisson equations for $u$ and $v$. In that case nested dissection gives connections between all the variables surrounding a domain. So the matrices $A_{11}, A_{12}$, and $A_{13}$ are half full (no couplings between $u$ and $v$ ). As most pressures are eliminated with the interior velocities, the matrices become dense.

Remark 2 In practice, $u$ and $v$ nodes on a separator may connect to the $P$ nodes with reversed signs. To ensure robustness we apply separate transforms to each velocity component.

Remark 3 Choosing a Householder transformation may seem arbitrary and not related to the physics of the problem. We may indeed choose other orthogonal transformations with the same effect (some alternatives are proposed at the end of section 4.4). The key is that one of the columns of $H$ - up to a normalizing factor - should be the vector $e$ with all entries equal to one. This yields the sum of all the fluxes through the interface, so there will be a new variable that represents the entire flux through the interface. The other new variables represent fluxes through the interface that are on average zero.

Remark 4 Instead of scaling the vectors in $H$ to unit length, we scale them to the length $m=\|e\|_{2}$ of the vector $e$ defining $H$. In that case the inverse of $H$ is $\frac{1}{m} H^{T}$.

Remark 5 Although not necessary for the decoupling process, we also apply an orthogonal transformation to $V$-nodes that are not coupled to a $P$-node in the first place. This is important for the dropping strategy proposed in the next section.

The situation depicted in eq. 3 now only occurs once per separator and velocity component, namely for the $V$-node still coupled to the $P$-nodes. Because of the transformation $\hat{b}$ is now zero, and no fill is generated.

So far we have not made any approximations, and while we have zeroed out most of the $V$-node/ $P$-node couplings, a dropping strategy has to be applied in the $V-V$ part to get a sparse preconditioner for the Schur-complement. However, the Householder transformation combined with standard dropping techniques for the SPD case will generally not lead to grid independent convergence. This requires that the approximation is spectrally equivalent to the original matrix. We will consider a new way of dropping in the next section which has this property. 


\subsection{Dropping strategy}

The general idea of the approximation is the following. We replace the flux through grid cell faces forming a separator by the combined flux through that separator (see Remark 3 in the previous section). Then we try to reduce the problem of finding all separator velocities by dropping and elimination to the related problem of finding the new fluxes (or summed velocities). This reduced problem can still be understood in terms of conservation of mass and momentum and its form is very similar to the original problem.

Let us consider an orthogonal operator that is more intuitive than the Householder transformation. Suppose $e$ is a vector with all ones and $C$ is an orthogonal extension of $e$ such that the length of every column is the same. Define a square matrix

$$
H=[C, e],
$$

which is orthogonal up to a constant factor (see Remark 4 in the previous section). This operator is applied to the velocity component in normal direction on the separator. These velocities have the same sign for the connection to the pressure and therefore again only one row remains in $H^{T} B_{1}$. The first component of $H^{T} v$ will be the sum of the components of $v$; we will call this a $V_{\Sigma}$-node from now on. To develop some intuition, we first give a simple example of the dropping strategy which reveals that the resulting reduced problem can be viewed as a coarse representation of the original one. In section 4.4.2 we then perform a more general analysis.

\subsubsection{Example of dropping}

Consider the familiar tridiagonal matrix with elements $[-12-1]$ on the sub-, main, and superdiagonal, respectively, which arises when discretizing the 1D Laplace equation on a regular grid. We premultiply it by the block diagonal matrix $H$ with diagonal blocks

$$
\left[\begin{array}{cc}
-1 & 1 \\
1 & 1
\end{array}\right]
$$

and postmultiply by its transpose (the same matrix here). Every pair of rows of the transformed matrix has the form

$$
\begin{array}{cccccc}
1 & 1 & 6 & 0 & 1 & -1 \\
-1 & -1 & 0 & 2 & 1 & -1
\end{array} .
$$

Next we make an odd-even ordering for the unknowns (equivalent to shifting the $V_{\Sigma}$-nodes to the end of the matrix). This new matrix has the form

$$
\left[\begin{array}{ll}
A_{11} & A_{12} \\
A_{21} & A_{22}
\end{array}\right] .
$$

The matrix $A_{11}$ is tridiagonal with entries [ $\left.\begin{array}{lll}1 & 6 & 1\end{array}\right]$, and $A_{22}$ is tridiagonal with entries $[-12-1]$. So $A_{22}$ is a representation of the original problem on a twice 
as coarse grid (up to scaling). The blocks $A_{12}$ and $A_{21}$ have row sum zero, a typical row of $A_{12}$ being $\left[\begin{array}{lll}1 & 0 & -1\end{array}\right]$. We just drop these two blocks and take the remaining part as the approximation. The fact that both $A_{11}$ and $A_{22}$ are principle submatrices of the above matrix infers that they both are SPD, so the approximation does not lead to a singular or indefinite matrix. Note that the elements in the dropped part are quite big and would not be dropped by traditional drop-by-value strategies.

To prove grid-independent convergence when using the resulting matrix as preconditioner, we have to show that $A_{21} A_{11}^{-1} A_{12} \leq \gamma^{2} A_{22}$, for some $\gamma<1$ independent of the size of the matrix (the case $\gamma=1$ follows directly from the positiveness of the Schur-complement of the original problem. For gridindependence we just need some extra margin). We can apply Fourier analysis in this constant coefficient case, which leads to the problem of finding the maximum of

$$
\frac{\sin (\theta)^{2}}{(6+2 \cos (\theta)) \sin (\theta / 2)^{2}}=\frac{\cos (\theta / 2)^{2}}{1+\cos (\theta / 2)^{2}} .
$$

This amounts to finding the maximum of $x /(1+x)$ on $[0,1]$, which is a monotonic function, so the maximum is $1 / 2$.

Another approach is to view the matrix as a sum of "element" matrices $E_{i}$ and the preconditioner as a sum of $F_{i}$. Using the Rayleigh quotient, one can easily show that the condition number of the preconditioned matrix is bounded if $\left(x, E_{i} x\right) /\left(x, F_{i} x\right)$ is bounded from below and above for $x$ not in the common null space of $E_{i}$ and $F_{i}$ for all $i$ (e.g. see (Axelsson \& Larin 1997)). The singular vector of the transformed matrix (4) is $[0,1,0,1,0,1, \ldots]^{T}$, and for the element matrices of the transformed problem and the approximation we can use

$$
E_{i}=\left[\begin{array}{cc|cc}
3 & 1 & 1 & -1 \\
1 & 1 & 1 & -1 \\
\hline 1 & 1 & 3 & -1 \\
-1 & -1 & -1 & 1
\end{array}\right], \quad F_{i}=\left[\begin{array}{cc|cc}
3 & 0 & 1 & 0 \\
0 & 1 & 0 & -1 \\
\hline 1 & 0 & 3 & 0 \\
0 & -1 & 0 & 1
\end{array}\right]
$$

Both matrices are nonnegative and the condition number of $E_{2}^{-1} E_{1}$ is bounded on the space orthogonal to $[0,1,0,1]^{T}$. This approach also reveals that we can replace $A_{11}$ by any positive diagonal matrix and still have a condition number independent of the mesh size. This concludes our simple example.

\subsubsection{General analysis}

These contemplations suggest that the following lemma and its corollary play a key role in devising a dropping strategy:

Lemma 1 Principal submatrices of an (S)PD-matrix are (S)PD.

\section{Corollary 1}

$$
\text { If }\left[\begin{array}{ll}
A_{11} & A_{12} \\
A_{21} & A_{22}
\end{array}\right] \text { is (S)PD then }\left[\begin{array}{cc}
A_{11} & O \\
O & A_{22}
\end{array}\right] \text { is }(S) P D \text {. }
$$


Since we only make approximations in the $A$ part of the matrix $K$, we have the following lemma.

Lemma 2 If $A$ is $S P D$, the condition number of the preconditioned $K$ matrix is bounded by the condition number of the preconditioned $A$, where as preconditioner an SPD approximation of $A$ is used.

Proof: Consider the generalized eigenvalue problem

$$
\left[\begin{array}{cc}
A-\lambda \tilde{A} & (1-\lambda) B \\
(1-\lambda) B^{T} & O
\end{array}\right]\left[\begin{array}{l}
x_{1} \\
x_{2}
\end{array}\right]=0
$$

where $\tilde{A}$ denotes an SPD approximation of $A$. We see that for $\lambda \neq 1(\lambda=1$ is clearly an eigenvalue) we can scale the border by any constant. So the eigenvalue problem is in fact an eigenvalue problem restricted to the kernel of the divergence (or constraint) operator $B^{T}$. Suppose $Q$ is an orthogonal basis for the kernel of $B^{T}$, then we have to find the eigenvalues of the pencil $\left(Q^{T} A Q, Q^{T} \tilde{A} Q\right.$. Now

$$
\begin{aligned}
\lambda_{\min }(A, \tilde{A}) & =\min _{x} \frac{(x, A x)}{(x, \tilde{A} x)} \leq \min _{y} \frac{\left(y, Q^{T} A Q y\right)}{\left(y \cdot Q^{T} \tilde{A} Q y\right)} \leq \frac{\left(y, Q^{T} A Q y\right)}{\left(y \cdot Q^{T} \tilde{A} Q y\right)} \\
& \leq \max _{y} \frac{\left(y, Q^{T} A Q y\right)}{\left(y \cdot Q^{T} \tilde{A} Q y\right)} \leq \max _{x} \frac{(x, A x)}{(x, \tilde{A} x)}=\lambda \max (A, \tilde{A}) .
\end{aligned}
$$

Hence, the eigenvalues of the preconditioned $K$ are bounded by the eigenvalues of the preconditioned $A$, which leads to the result.

These lemmas set the ground for further reasoning that will lead to gridindependent convergence. In the remainder of this section we assume that $A$ is symmetric and positive definite. Let us extend $H$ with an identity for the unknowns that are not transformed and write $H=\left[H_{1}, H_{2}\right]$, where

$$
H_{1}=\left[\begin{array}{l}
C \\
0
\end{array}\right], \quad H_{2}=\left[\begin{array}{ll}
e & 0 \\
0 & I
\end{array}\right]
$$

The transformed matrix is given by

$$
H^{T} A H=\left[\begin{array}{ll}
H_{1}^{T} A H_{1} & H_{1}^{T} A H_{2} \\
H_{2}^{T} A H_{1} & H_{2}^{T} A H_{2}
\end{array}\right] .
$$

Here $H_{2}^{T} A H_{2}$ is a Galerkin approximation of $A$ and hence it can be viewed as a discretization on a coarser grid (in fact it is an aggregation similar to that used by Notay (2010), albeit Notay applies the aggregation directly to the discretized PDE whereas we apply it to its Schur complement on the separators). If $A$ is obtained from a stable discretization of a second-order differential operator, then $H_{1}^{T} A H_{1}$ has a condition number independent of the mesh size if the dimension of $C$ is fixed (i.e. if the length of the separator is fixed). We will prove this for a very simple case using finite element theory. 
We just consider the operator $\frac{d}{d s}\left(p(s) \frac{d}{d s} \cdot\right)$ with $p>0$ on the interval $(0, d)$ with homogeneous Neumann boundary conditions. Hence the related bilinear form is $a(u, v)=\left(p u^{\prime}, v^{\prime}\right)=\int_{0}^{d} p u^{\prime} v^{\prime} d s$, where we have used the inner product $(u, v)=\int_{0}^{d} u v d s$. The norm associated with this inner product is denoted by $\|\cdot\|$. Here $u, v$ and $p$ are all functions in the Sobolev space $\mathcal{H}_{1}(0, d)$, which consists of all continuous functions that are piecewise differentiable. We will also apply this inner product to vectors of functions, which should be read as applying it element by element.

Lemma 3 Let $A=a(V, V)$ and $M=(V, V)$, where $V=\left[\phi_{1}(s), \phi_{2}(s), \ldots \phi_{N}(s)\right]$ is a row vector of basis functions in $\mathcal{H}_{1}(0, d)$ with the property that there exists a constant $c$ such that $\frac{c}{h^{2}} M-\left(V^{\prime}, V^{\prime}\right)$ is nonnegative. If $c_{2}\left\|u^{\prime}\right\|^{2} \geq a(u, u) \geq$ $c_{1}\left\|u^{\prime}\right\|^{2}$, the spectral condition number of $\left(H_{1}^{T} A H_{1}\right) /\left(H_{1}^{T} M H_{1}\right)$ is bounded by $(d / h)^{2}$.

Proof: A straightforward substitution of $u=V H_{1} x$ in the inequality leads to

$$
c_{2}\left\|V^{\prime} H_{1} x\right\|^{2} \geq a\left(V H_{1} x, V H_{1} x\right)=\left(x, H_{1}^{T} A H_{1} x\right) \geq c_{1}\left\|V^{\prime} H_{1} x\right\|^{2} .
$$

Now the minimum of $\left\|f^{\prime}\right\| /\|f\|$, where $f$ is an arbitrary $\mathcal{H}_{1}$ function orthogonal to the constant function is just the eigenfunction of the 1D Laplace operator with homogeneous Neumann boundary conditions orthogonal to the constant, which is $\cos (\pi s / d)$. So

$$
\min _{x} \frac{\left\|V^{\prime} H_{1} x\right\|^{2}}{\left\|V H_{1} x\right\|^{2}} \geq \min _{f} \frac{\left\|f^{\prime}\right\|^{2}}{\|f\|^{2}}=\left(\frac{\pi}{d}\right)^{2} .
$$

Hence, the smallest eigenvalue of $\left(H_{1}^{T} A H_{1}\right) /\left(H_{1}^{T} M H_{1}\right)$ is bounded away from zero by $c_{1}\left(\frac{\pi}{d}\right)^{2}$. Now let us try to find an upper bound which is less than infinity. This maximum possible is related to the highest frequency we can build from $V H_{1} x$ such that the norm of $V^{\prime} H_{1} x$ becomes maximal. The shortest wave that can be represented is related to the mesh size $h$. Thus we came to the assumption in the theorem which can be quite easily verified in a special case using for instance the Gershgorin circle theorem. We find that

$$
\frac{\left\|V^{\prime} H_{1} x\right\|^{2}}{\left\|V H_{1} x\right\|^{2}} \leq \frac{c}{h^{2}} \frac{x^{T} H_{1}^{T} M H_{1} x}{\left\|V H_{1} x\right\|^{2}}=\frac{c}{h^{2}},
$$

so the spectral condition number of $H_{1}^{T} A H_{1} / H_{1}^{T} M H_{1}$ is bounded by $\frac{c c_{2} d^{2}}{c_{1} \pi h^{2}}$.

The constants $c_{1}$ and $c_{2}$ are easily determined, we can simply take the minimum and maximum of the function $p(s)$ on the interval, respectively. Although this lemma is based on a second-order differential operator, one could in fact find a similar statement for nonnegative operators with pseudo derivative $2 \nu$, where $\nu$ may be any positive real number. Such an operator is found, for instance, when writing down the continuous equations at the separators, leading to the so-called Steklov-Poincaré operator (see (Toselli \& Widlund 2005)). To return to our discussion, for $d$ in the lemma one could think of the length of 
the separator. So if $d$ decreases proportional with $h$ when refining the grid, the condition number of $H_{1}^{T} A H_{1}$ is bounded independently of the mesh size assuming we can bound the condition number of $M$ beforehand. The latter matrix is usually strictly diagonally dominant. So simply applying Gershgorin's theorem makes the assumption valid.

Now assume we have the following strengthened Cauchy-Schwarz inequality (Axelsson 1994, section 9.1)

$$
\left|x^{T} H_{1}^{T} A H_{2} y\right| \leq \gamma\left\{\left(x^{T} H_{1}^{T} A H_{1} x\right)\left(y^{T} H_{2}^{T} A H_{2} y\right)\right\}^{\frac{1}{2}}
$$

holding independently of the mesh size. In our case $H_{1}^{T} H_{2}=0$, and if the columns of $H_{1}$ or $H_{2}$ span an invariant subspace of $A$, then also $H_{1}^{T} A H_{2}=0$, hence $\gamma=0$. The latter is only approximately the case here, so we will find some $\gamma<1$. Lemma 9.2 from (Axelsson 1994) states that

$$
H_{2}^{T} A H_{1}\left(H_{1}^{T} A H_{1}\right)^{-1} H_{1}^{T} A H_{2} \leq \gamma^{2} H_{2}^{T} A H_{2},
$$

where the inequality should be understood in the sense that the sum of the left-hand side and the right-hand side gives a non-negative matrix. For ease of notation we write the transformed matrix (6) as

$$
\left[\begin{array}{ll}
A_{11} & A_{12} \\
A_{21} & A_{22}
\end{array}\right] \text {. }
$$

In this notation the above property reads $A_{21} A_{11}^{-1} A_{12} \leq \gamma^{2} A_{22}$. Now the preconditioner obtained by dropping $A_{21}$ and $A_{12}$ is SPD according to Corollary 1 The eigenvalues of the preconditioned $A$ matrix can be found from the following generalized eigenvalue problem.

$$
\left[\begin{array}{cc}
(1-\lambda) A_{11} & -\lambda A_{12} \\
-\lambda A_{21} & (1-\lambda) A_{22}
\end{array}\right]\left[\begin{array}{l}
x_{1} \\
x_{2}
\end{array}\right]=0
$$

which leads to $\left((1-\lambda)^{2} A_{22}-\lambda^{2} A_{21} A_{11}^{-1} A_{12}\right) x_{2}=0$ for $\lambda \neq 1$. Combined with the previous, we can only find eigenvalues for $(1-\lambda)^{2}<(\lambda \gamma)^{2}$, so $1-\lambda \gamma<$ $\lambda<1+\lambda \gamma$. So we find that the condition number of the preconditioned matrix is less than $(1+\gamma) /(1-\gamma)$, where $\gamma$ is independent of the mesh size. Using Lemma 2 we find the main result of this paper.

Theorem 5 If a strengthened Schwarz-inequality (7) holds for $0 \leq \gamma<1$ independent of the mesh size, then we have convergence independent of the mesh-size when the dropping process as discussed above is applied. The condition number of the preconditioned $K$ matrix is bounded by $(1+\gamma) /(1-\gamma)$.

The situation above remains the same if we apply the transformation to all separators at once. After the transformation, only the unknowns associated with $A_{22}$ are coupled to pressures. We may still have couplings between various separators in $A_{11}$, but the condition number of that matrix is independent of the mesh size. To lower the computational cost we also drop couplings between separators in $A_{11}$. We conclude this section by a number of remarks concerning the dropping strategy. 
Scalar equations. The reader may have noticed that in this section we hardly mentioned the pressure. In fact, the combination of orthogonal transformations and dropping may also be applied to the pure diffusion problem. In section 5 we will start out by showing numerical results for the scalar Poisson equation.

The nonsymmetric case. One may ask how much of the above can be generalized to the nonsymmetric case (for instance the Navier-Stokes equations). Assume the nonsymmetric matrix $A$ is positive definite (PD), i.e. $(x, A x)>0$ for any non-trivial $x$. Then the Schur complement is PD and the orthogonal transformation does not destroy that property. Since all principle submatrices of a PD matrix are PD, the approximation will be PD. So the factorization will not break down. To say something about the condition number of the preconditioned matrix is more difficult. For a mild deviation from symmetry we expect the same behavior as for the symmetric case. However, the numerical results for the Navier-Stokes equations at relatively high Reynolds-numbers indicate that the method works very well even for highly non-symmetric matrices.

Numerical stability. In traditional lumping, only possible for M-matrices, one simply lumps a coefficient on the diagonal. This means that a nonnegative matrix is subtracted. Eijkhout (Eijkhout 1992) showed already in the nineties that this may give a zero on the diagonal. This is easy to preclude by simply not allowing the diagonal to become zero. What is much harder to prevent is the occurrence of independent systems in the preconditioner, some of which may be singular. This easily occurs in anisotropic problems. The proposed dropping does not suffer from these problems.

Alternatives for $\mathbf{H}$. Finally we propose a simple orthogonal extension to $e$ in order to form $H$. Let $m$ be the order of $H$ and note that $[1,-1,0, \cdots, 0]^{T}$, $[1,1,-2,0, \cdots, 0]^{T}, \cdots,[1, \cdots, 1,-(m-1)]^{T}, e$ are all orthogonal. They can be used for the extension after a proper scaling to the length of $e$. The application of this operator can be implemented by keeping a partial sum. In this way about $2 m$ additions of rows of the matrix it is applied to are needed. The Householder transform has a similar operation count. One may ask whether alternative choices for $C$ in $H_{1}$ influence the convergence. This is not the case. We can replace $H_{1}$ by $H_{1} Q$. For arbitrary orthogonal matrices $Q$ this has no influence on (7/8) and the following analysis.

\subsection{Iteration in the kernel of $B^{T}$}

Since the fill of the $B$ part remains at most 2 per row during the whole process, we will not drop there. This means that the $B$ matrix is exact in the factorization, and with appropriate dropping (such as the strategy introduced in the previous section), the eigenvalues of the preconditioned matrix will all be positive and real. Still, we cannot directly apply the preconditioned conjugate 
gradient method since for that both original and preconditioner must be positive definite in the Krylov subspace. We can enforce this condition by building the Krylov subspace $\mathcal{K}\left(\tilde{K}^{-1} K, x\right)$ on a starting solution $x$ that satisfies the constraint. In exact arithmetic $\mathcal{K}$ then remains in the kernel of $B^{T}$. In practice, accumulation of round-off errors will undermine this property.

This problem is often encountered in the field of constraint optimization, and Gould et al. (2001) have developed a variant of the conjugate gradient method, Projected Preconditioned CG (PPCG), which can be used for the Stokes prob-

lem. There are various ways to find a particular solution of $B^{T} v=b_{2}$, one of which is solving the system once, replacing $K$ by the preconditioner.

For the Navier-Stokes equations one could devise a Projected Preconditioned FOM method, as long as the eigenvalues of the preconditioned matrix are in the right half plane, but for the results shown in section 5.4 we simply used MATLAB's gmres.

\subsection{Program structure}

Before looking at numerical results, let us review the complete algorithm and remark on some implementation issues. The main structure of the program is as follows

1. Perform a domain decomposition on $F(A) \cup F(B) F(B)^{T}$. We just make a rectangular decomposition of the domain here.

2. Group the variables into subdomain variables and separator variables (velocities connecting to variables in more than one subdomain). All pressures are treated as subdomain variables at this stage.

3. Group the separator variables according to variable type (i.e. $u, v$ ) and the subdomains they have connections to. Thus, we will get a group of $u$-velocities connecting to variables on subdomains 1 and 2 , for instance.

4. In the corners of subdomains a complete conservation cell (see fig. 1) can occur on the separators. This would lead to a singularity in step 6 The velocities making up such a cell are flagged ' $V_{\Sigma}$ '-nodes (cf. step 8). Both these $V_{\Sigma}$-nodes and the $P$-node in the cell will be retained in the Schurcomplement.

5. Pick for every domain a $P$-node to be kept in the reduction, and shift these to the end of the ordering (i.e. retain them in the Schur-complement).

6. Eliminate all interior variables of the subdomains and construct the Schur complement system for the velocity separators and the selected pressures.

7. Perform the transformation on each separator group identified in step 3 .

8. Identify $V_{\Sigma}$ nodes (separator velocities that still connect to two pressures) and put them at the end of the ordering, just before the remaining pressure nodes. 
9. Drop all connections between non- $V_{\Sigma}$ nodes and $V_{\Sigma}$ nodes, and between non- $V_{\Sigma}$ nodes in different separator groups. The resulting matrix is blockdiagonal with the 'reduced Schur-complement' in the lower right corner.

10. Iterate on the Schur complement using the matrix of the previous step as preconditioner. This preconditioner is easily applied using LU decompositions of all non- $V_{\Sigma}$ blocks and the reduced system.

In three space dimensions, step 3 is implemented by first numbering the faces, then the edges and then the corners of the box-shaped subdomains. We note that this is a special case of the hierarchical interface decomposition (HID) used by Hénon \& Saad (2006) and Gaidamour (2008).

\subsection{Computational complexity}

We will now discuss the complexity of the algorithm, implemented as discussed in the previous section. We assume that a direct method with optimal complexity is used for the solution of the relevant linear systems, so in 3D if the number of unknowns is $\mathcal{O}(N)$, the work is $\mathcal{O}\left(N^{2}\right)$, as with Nested Dissection. For the 3D (Navier-)Stokes equations, we have $N=\mathcal{O}\left(n^{3}\right)$ unknowns, where $n$ is the number of grid cells in one space dimension. We keep the subdomain size constant and denote the number of unknowns per subdomain by $S=\mathcal{O}\left(s^{3}\right)$ (here $s$ is the fixed separator length). Hence, there will be $N / S$ subdomains. Per domain there will be $\mathcal{O}\left(s^{2}\right)$ non- $V_{\Sigma^{-}}$and $\mathcal{O}(1) V_{\Sigma}$-nodes. Per domain the amount of work required is as follows:

1. $\mathcal{O}\left(S^{2}\right)$ for the subdomain elimination;

2. transformation on faces with $H: \mathcal{O}\left(s^{4}\right)$;

3. factorization of non- $V_{\Sigma}$ nodes: $\mathcal{O}\left(\left(s^{2}\right)^{3}\right)=O\left(S^{2}\right)$.

The total over all domains is $\mathcal{O}(N / S) \mathcal{O}\left(S^{2}\right)=\mathcal{O}(N S)$, so in this part the number of operations decreases linearly with $S$ (e.g. by a factor 8 if $s$ is halved).

The solution of the reduced problem $\left(V_{\Sigma}\right.$-nodes) requires $\mathcal{O}\left((N / S)^{2}\right)$ operations. Here doubling $s$ will decrease the work by a factor 64 . So in total the work per iteration is $\mathcal{O}(N S)+\mathcal{O}\left((N / S)^{2}\right)$. The number of iterations is constant for $S$ constant. There is, however, a positive dependence on $S$ as we may expect. In the next section we will observe that the number of iterations is proportional to $\log (S)$. So if we double $s$, a fixed amount of iterations is added.

It is clear that if we solved the reduced problem iteratively by applying our method recursively until the problem has a fixed grid-independent size, the overall complexity would be $\log (S) \mathcal{O}(N S)$.

\section{Numerical experiments}

In this section we will demonstrate the performance of the new solver by applying it to a series of increasingly complex problems relevant to computational 
fluid dynamics. For each problem we first keep the subdomain size constant while refining the mesh. As discussed in the previous section, the complexity of the algorithm will then be linear in the number of unknowns except when solving the reduced Schur complement: the operations required to factor a single subdomain matrix stays the same and the number of subdomains increases linearly with the grid size. Furthermore, both size and connectivity pattern of the separators remain the same so the amount of work per separator remains constant while the number of separators increases linearly, too.

The second experiment will be to fix the grid size and vary the subdomain size (i.e. the number of subdomains). The expectation here is that due to fill-in the bulk of the work load shifts from the Schur-complement towards the subdomain factorization as the size of the subdomains is increased.

For each experiment, the following data is displayed:

- $n_{x}$ - the grid size is $n_{x} \times n_{x}\left(n_{x} \times n_{x} \times n_{x}\right)$ in 2D (3D), respectively.

- $s_{x}$ - the subdomain size is $s_{x} \times s_{x}\left(s_{x} \times s_{x} \times s_{x}\right)$ in $2 \mathrm{D}(3 \mathrm{D})$, respectively.

- N - number of unknowns (size of the saddle point matrix),

- nnz - number of nonzeros in original matrix,

- $\mathrm{N}_{\mathrm{S}}$ - number of unknowns on the separators and remaining p's (size of the Schur-complement),

- n - number of $V_{\Sigma}^{\prime} s$ and remaining p's (size of reduced Schur-complement),

- iter - number of CG iterations performed on the Schur-complement to reduce the residual norm by $1 /$ tol $=10^{8}$,

- fill 1 - grid-independent part of relative fill-in (number of nonzeros in the solver divided by number of nonzeros in original matrix). The gridindependent portion consists of

- a) fill-in generated while factoring the subdomain matrices

- b) fill-in generated while constructing the Schur-complement

- c) fill-in generated while factoring the separator-blocks of the preconditioner

- fill 2 - grid-dependent part of relative fill-in, generated when factoring the $n \times n$-dimensional reduced Schur-complement.

- $\kappa$ - condition estimate of the preconditioned Schur-complement: fraction of the largest and smallest eigenvalue (by magnitude) of the generalized eigenvalue problem $\mathrm{S} x+\lambda \mathrm{M} x=0$, where $\mathrm{S}$ is the Schur-complement, and $\mathrm{M}$ the preconditioner used. We use approximations to the actual eigenvalues computed by MATLAB's 'eigs' command (Not all tables contain this value). 
Remark the fill listed under fill $1 \mathrm{~b}$ ) can be avoided by not explicitly constructing the Schur-complement. The fill listed as 'fill 2' grows with increasing grid size, but it can be made grid-independent by solving $S_{2}$ iteratively, too (i.e. by applying our method recursively).

We do not show plots of the convergence behavior. Since all the results are obtained by CG the convergence is, apart from the first few digits gained, completely regular, which shows that the eigenvalues, except for a few outliers at the beginning, appear in a cluster. The relatively stringent convergence tolerance of 8 digits ensures that the overall convergence behavior does not strongly depend on the choice of the initial vector. Choosing a smaller tolerance results in stagnation for some of the tests below because the conditioning of the matrix doesn't allow for more accurate solutions.

The general behavior we observe in the second experiment is that the number of iterations scales with $\log \left(s_{x}\right)$, where $s_{x}$ is the separator length. So doubling the separator length means an increase of the number of iterations by a constant amount.

\subsection{The Poisson equation}

We first investigate Poisson's equation, discretized using second order central differences on a regular structured grid (standard 5-point and 7-point stencils in $2 \mathrm{D}$ and 3D, respectively). This is an important case as solving Poisson's equation is central to most CFD problems, for instance to determine the pressure in explicit time stepping algorithms. Tables 1 and 2 show the $2 \mathrm{D}$ results. The first shows the dependence on grid refinement and the latter the influence of the domain sizes. Similar results for the 3D case are shown in tables 3 and 4 .

\begin{tabular}{|c|r|r|r|r|r|r|r|r|}
\hline$n_{x}$ & $\mathrm{~N}$ & $\mathrm{nnz}$ & $\mathrm{N}_{\mathrm{S}}$ & $\mathrm{n}$ & iter & fill 1 & fill 2 & $\kappa$ \\
\hline 32 & 1024 & 5112 & 240 & 48 & 21 & 5.53 & 0.20 & 7.04 \\
64 & 4096 & 20472 & 960 & 192 & 21 & 5.52 & 0.39 & 7.04 \\
128 & 16384 & 81912 & 3840 & 768 & 21 & 5.52 & 0.68 & 7.04 \\
256 & 65536 & 327672 & 15360 & 3072 & 21 & 5.52 & 1.03 & 7.04 \\
512 & 262144 & 1310712 & 61440 & 12288 & 21 & 5.52 & 1.59 & 7.04 \\
1024 & 1048576 & 5242872 & 245760 & 49152 & 21 & 5.52 & 2.20 & 7.04 \\
\hline
\end{tabular}

Table 1: 2D Poisson-equation - grid refinement, subdomain size $s_{x}=8$.

\subsection{Darcy's law}

For flows in porous media one often has to solve the Darcy problem, where $A$ is just a diagonal matrix. One approach is to eliminate the velocities, which leads to a Poisson equation. Care has to be taken when calculating the velocities, because the gradient operator has to be applied to the pressure. In this numerical 


\begin{tabular}{|c|r|r|r|r|r|r|r|r|}
\hline$s_{x}$ & $\mathrm{~N}$ & $\mathrm{nnz}$ & $\mathrm{N}_{\mathrm{S}}$ & $\mathrm{n}$ & iter & fill 1 & fill 2 & $\kappa$ \\
\hline 4 & 1048576 & 5242872 & 458752 & 196608 & 16 & 2.01 & 11.5 & 4.00 \\
8 & 1048576 & 5242872 & 245760 & 49152 & 21 & 5.52 & 2.29 & 7.04 \\
16 & 1048576 & 5242872 & 126976 & 12288 & 27 & 9.84 & 0.39 & 11.2 \\
32 & 1048576 & 5242872 & 64512 & 3072 & 32 & 13.8 & 0.063 & 16.5 \\
\hline
\end{tabular}

Table 2: 2D Poisson-equation - increasing subdomain size, grid-size $n_{x}=1024$

\begin{tabular}{|r|r|r|r|r|r|r|r|r|}
\hline$n_{x}$ & $\mathrm{~N}$ & $\mathrm{nnz}$ & $\mathrm{N}_{\mathrm{S}}$ & $\mathrm{n}$ & iter & fill 1 & fill 2 & $\kappa$ \\
\hline 16 & 4096 & 28660 & 1352 & 56 & 24 & 29.7 & 0.064 & 10.1 \\
32 & 32768 & 229364 & 10816 & 448 & 25 & 29.0 & 0.36 & 10.2 \\
64 & 262144 & 1834996 & 86528 & 3584 & 25 & 29.0 & 1.53 & - \\
\hline
\end{tabular}

Table 3: 3D Poisson-equation - grid refinement, subdomain size $s_{x}=8$

\begin{tabular}{|c|r|r|r|r|r|r|r|r|}
\hline$s_{x}$ & $\mathrm{~N}$ & $\mathrm{nnz}$ & $\mathrm{N}_{\mathrm{S}}$ & $\mathrm{n}$ & iter & fill 1 & fill 2 & $\kappa^{\star}$ \\
\hline 4 & 262144 & 1834996 & 151552 & 28672 & 19 & 3.68 & 52.0 & 5.75 \\
8 & 262144 & 1834996 & 86528 & 3584 & 25 & 29.0 & 1.5 & 10.2 \\
16 & 262144 & 1834996 & 46144 & 448 & 30 & 116.2 & 0.045 & 16.7 \\
\hline
\end{tabular}

Table 4: 3D Poisson-equation - increasing subdomain size, grid size $n_{x}=64$.

$\star$ Computed at $n_{x}=32$.

differentiation of the pressure field, round-off errors may be amplified too much to obtain an accurate solution. Therefore, Darcy's problem is often solved in primitive form. Tables 5 through 8 show the numerical results for Darcy's law in two and three space dimensions.

\begin{tabular}{|c|r|r|r|r|r|r|r|r|}
\hline$n_{x}$ & $\mathrm{~N}$ & $\mathrm{nnz}$ & $\mathrm{N}_{\mathrm{S}}$ & $\mathrm{n}$ & iter & fill 1 & fill 2 & $\kappa$ \\
\hline 16 & 736 & 2400 & 65 & 17 & 16 & 5.53 & 0.061 & 3.77 \\
32 & 3008 & 9920 & 385 & 109 & 25 & 6.29 & 0.24 & 10.8 \\
64 & 12160 & 40320 & 1793 & 533 & 26 & 6.65 & 0.49 & 12.2 \\
128 & 48896 & 162560 & 7681 & 2341 & 26 & 6.82 & 1.00 & 12.6 \\
256 & 196096 & 652800 & 31745 & 9797 & 26 & 6.91 & 1.69 & 12.6 \\
512 & 785408 & 2616320 & 129025 & 40069 & 26 & 6.95 & 2.64 & 12.7 \\
1024 & 3143680 & 10475520 & 520193 & 162053 & 26 & 6.97 & 3.58 & - \\
\hline
\end{tabular}

Table 5: 2D Darcy-equation - grid refinement, subdomain size $s_{x}=8$

\begin{tabular}{|c|r|r|r|r|r|r|r|r|}
\hline$s_{x}$ & $\mathrm{~N}$ & $\mathrm{nnz}$ & $\mathrm{N}_{\mathrm{S}}$ & $\mathrm{n}$ & iter & fill 1 & fill 2 & $\kappa$ \\
\hline 8 & 3143680 & 10475520 & 520193 & 162053 & 26 & 6.97 & 3.58 & 12.7 \\
16 & 3143680 & 10475520 & 258049 & 40069 & 29 & 11.1 & 0.66 & 17.6 \\
\hline
\end{tabular}

Table 6: 2D Darcy-equation - increasing subdomain size, grid size $n_{x}=512$ 


\begin{tabular}{|c|r|r|r|r|r|r|r|r|}
\hline$n_{x}$ & $\mathrm{~N}$ & $\mathrm{nnz}$ & $\mathrm{N}_{\mathrm{S}}$ & $\mathrm{n}$ & iter & fill 1 & fill 2 & $\kappa$ \\
\hline 8 & 1856 & 6720 & 492 & 171 & 34 & 10.8 & 1.28 & 14.0 \\
16 & 15616 & 57600 & 5878 & 2683 & 36 & 10.2 & 17.6 & 15.3 \\
32 & 128000 & 476160 & 54762 & 27819 & 36 & 9.73 & 87.7 & 15.4 \\
40 & 251200 & 936000 & 109972 & 56971 & 36 & 9.65 & 167. & - \\
\hline
\end{tabular}

Table 7: 3D Darcy-equation - grid refinement, subdomain size $s_{x}=4$

\begin{tabular}{|c|r|r|r|r|r|r|r|r|}
\hline$s_{x}$ & $\mathrm{~N}$ & $\mathrm{nnz}$ & $\mathrm{N}_{\mathrm{S}}$ & $\mathrm{n}$ & iter & fill 1 & fill 2 & $\kappa^{\star}$ \\
\hline 4 & 251200 & 936000 & 109972 & 56971 & 36 & 9.65 & 167. & 15.4 \\
8 & 251200 & 936000 & 53037 & 11601 & 39 & 50.2 & 16.7 & 18.3 \\
\hline
\end{tabular}

Table 8: 3D Darcy-equation - increasing subdomain size, grid size $n_{x}=40$. $\star$ Computed at $n_{x}=32$.

\subsection{A Stokes problem}

The problem is a two-dimensional Stokes equation on the unit square

$$
\left.\begin{array}{rl}
-\nu \Delta \mathbf{u}+\nabla p & =0, \\
\nabla \cdot \mathbf{u} & =0
\end{array}\right\}
$$

where $\mathbf{u}(x, y)$ is the velocity field and $p(x, y)$ the pressure field; the parameter $\nu$ controls the amount of viscosity. We can get rid of the parameter $\nu$ by defining a new pressure variable $\bar{p}=p / \nu$. If the first equation is divided by $\nu$, we can substitute $p$ by $\bar{p}$ and the parameter $\nu$ is gone. So we may assume that $\nu=1$.

These equations are discretized on a uniform staggered grid (a C-grid, see fig. 11) which results in an $\mathcal{F}$-matrix. It is singular because the pressure field is determined up to a constant.

For the Stokes problem the matrix $B^{T}$ represents the discrete divergence operator. Consequently, we call the kernel of this matrix the divergence free space. As a solution of this problem we choose a random vector in the divergence free space. So the right-hand side of the divergence equation is zero in our case.

We start off the iteration with the zero vector (which is trivially in the divergence free space) and therefore we can use the projected conjugate gradient method (see section 4.5). Results are summarized in tables 9 through 12 ,

\begin{tabular}{|c|r|r|r|r|r|r|r|r|}
\hline$n_{x}$ & $\mathrm{~N}$ & $\mathrm{nnz}$ & $\mathrm{N}_{\mathrm{S}}$ & $\mathrm{n}$ & iter & fill 1 & fill 2 & $\kappa$ \\
\hline 16 & 736 & 4196 & 65 & 17 & 18 & 7.79 & 0.057 & 4.93 \\
32 & 3008 & 17604 & 385 & 109 & 27 & 8.39 & 0.25 & 12.8 \\
64 & 12160 & 72068 & 1793 & 533 & 31 & 8.68 & 0.65 & 13.8 \\
128 & 48896 & 291588 & 7681 & 2341 & 31 & 8.72 & 1.33 & 14.2 \\
256 & 196096 & 1172996 & 31745 & 9797 & 31 & 8.70 & 2.40 & 14.6 \\
512 & 785408 & 4705284 & 129025 & 40069 & 31 & 8.60 & 3.83 & 15.0 \\
\hline
\end{tabular}

Table 9: 2D Stokes-equation - grid refinement, subdomain size $s_{x}=8$ 


\begin{tabular}{|c|r|r|r|r|r|r|r|r|}
\hline$s_{x}$ & $\mathrm{~N}$ & $\mathrm{nnz}$ & $\mathrm{N}_{\mathrm{S}}$ & $\mathrm{n}$ & iter & fill 1 & fill 2 & $\kappa$ \\
\hline 4 & 785408 & 4705284 & 260097 & 162053 & 24 & 3.65 & 20.0 & 9.6 \\
8 & 785408 & 4705284 & 129025 & 40069 & 31 & 8.60 & 3.83 & 15.0 \\
16 & 785408 & 4705284 & 63489 & 9797 & 38 & 15.7 & 0.60 & 21.9 \\
\hline
\end{tabular}

Table 10: 2D Stokes-equation - increasing subdomain size, grid size $n_{x}=512$

\begin{tabular}{|c|r|r|r|r|r|r|r|r|}
\hline$n_{x}$ & $\mathrm{~N}$ & $\mathrm{nnz}$ & $\mathrm{N}_{\mathrm{S}}$ & $\mathrm{n}$ & iter & fill 1 & fill 2 & $\kappa$ \\
\hline 8 & 1856 & 13728 & 492 & 171 & 34 & 13.9 & 1.20 & 16.6 \\
16 & 15616 & 122304 & 5878 & 2683 & 41 & 12.5 & 16.4 & 23.8 \\
32 & 128000 & 1029504 & 54762 & 27819 & 43 & 11.5 & 103. & 27.1 \\
40 & 251200 & 2030880 & 109972 & 56971 & 43 & 11.3 & 168. & - \\
\hline
\end{tabular}

Table 11: 3D Stokes-equation - grid refinement, subdomain size $s_{x}=4$

\begin{tabular}{|c|r|r|r|r|r|r|r|r|c|}
\hline$s_{x}$ & $\mathrm{~N}$ & $\mathrm{nnz}$ & $\mathrm{N}_{\mathrm{S}}$ & $\mathrm{n}$ & iter & fill 1 & fill 2 & $\kappa^{\star}$ \\
\hline 4 & 251200 & 2030880 & 109972 & 56971 & 43 & 11.3 & 167. & 27.1 \\
8 & 251200 & 2030880 & 53037 & 11601 & 49 & 65.8 & 12.1 & 39.1 \\
\hline
\end{tabular}

Table 12: 3D Stokes-equation - increasing subdomain size, grid size $n_{x}=40$.

$\star$ Computed at $n_{x}=32$.

\subsection{Incompressible flow in a lid-driven cavity}

As test problem for the Navier-Stokes equations we use the lid driven cavity. In (Tiesinga et al. 2002) this problem was studied near the transition point from steady to transient flow. The stability of steady and periodic solutions was investigated using the Newton-Picard method (Lust et al. 1999) with the $\theta$-method for time stepping (with $\theta$ slightly larger than 0.5 in order to damp high-frequency modes which would otherwise show up as spurious eigenvalues near the imaginary axis). The linear systems that have to be solved have a slightly increased diagonal, which improves the conditioning somewhat. The MRILU preconditioner (Botta \& Wubs 1999) used at the time converged slowly and not at a grid-independent rate. In a recent review (Elman et al. 2008), the performance of a number of block multi-level preconditioners is investigated for the steady problem for Reynolds numbers up to 1000. These methods also solve the coupled equations, but perform inner iterations on the velocity and pressure part separately and hence require many parameters to be tuned. Below we demonstrate robust, grid-independent convergence for the driven cavity problem at Reynolds-numbers of up to 8000.

The problem consists of calculating the flow in a square cavity with uniformly moving lid. The domain and boundary conditions of the lid-driven cavity problem are shown in fig. 3, where $u$ and $v$ denote the velocity in $x$ - and $y$-direction, respectively. 


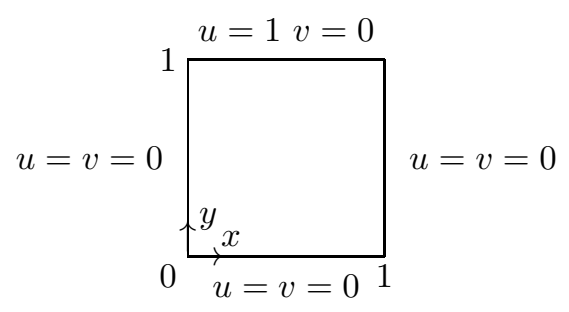

Figure 3: Geometry for the lid-driven cavity problem.

The equations are given by

$$
\left.\begin{array}{rl}
-\mathbf{u} \cdot \nabla \mathbf{u}+\frac{1}{R e} \Delta \mathbf{u}-\nabla p & =0 \\
\nabla \cdot \mathbf{u} & =
\end{array}\right\}
$$

For the discretization we use a symmetry-preserving space discretization (Verstappen \& Veldman 2003), which is stable and does not introduce artificial diffusion. Furthermore, the grid is stretched towards the boundaries in order to resolve the boundary layers. The ratio between largest and smallest mesh size is about 5. This also means that we really need to change to fluxes through grid cell boundaries instead of velocities in order to get the required property that all elements in $B$ have the same magnitude (see the beginning of section 4). The convergence tolerance is set to $10^{-6}$ in these experiments. The system matrix is the Jacobian from the first step of the Newton method at the current Reynolds number. In order to avoid convergence problems of Newton's method, we use the result at the previous Reynolds-number as a starting solution (The Reynolds numbers used are shown in table 13).

We first focus on the effect of increasing the Reynolds-number (cf. table 13). The convergence is not independent of the Reynolds-number. In our view this is not surprising, because the underlying continuous problem changes with the Reynolds number and more and more eigenvalues are getting close to the origin. This is different from the dependence on the mesh, where the continuous problem stays the same and all eigenvalues near the origin stay at their place.

Next we refine the grid at a high Reynolds-number of 8000, close to the point (cf. Tiesinga et al. (2002)) where the steady state becomes unstable; results are shown in table 14. Note that the number of iterations is going down as we decrease the mesh-size. This is because with decreasing mesh-size the physical size of the subdomains is decreasing if we keep the number of unknowns per subdomain the same. As the physical subdomain decreases, the diffusion plays a more important role than the advection on that scale. Since the approximations take place at the subdomain scale, the convergence behavior tends to that of the Stokes problem.

We conclude by mentioning that with the resulting preconditioner it was also quite easy to compute eigenvalues using MATLAB's eigs routine (i.e. ARPACK). Hence we can now study the stability problem near the point where the steady state becomes unstable using eigenvalue analysis. 


\begin{tabular}{|c|r|r|r|r|r|r|c|}
\hline $\mathrm{Re}$ & $\mathrm{N}$ & $\mathrm{nnz}$ & $\mathrm{N}_{\mathrm{S}}$ & $\mathrm{n}$ & iter & fill 1 & fill 2 \\
\hline 500 & 785408 & 6794252 & 129025 & 40069 & 59 & 6.41 & 2.59 \\
1000 & 785408 & 6794252 & 129025 & 40069 & 73 & 6.39 & 2.59 \\
2000 & 785408 & 6794252 & 129025 & 40069 & 87 & 6.38 & 2.65 \\
4000 & 785408 & 6794252 & 129025 & 40069 & 104 & 6.35 & 2.78 \\
8000 & 785408 & 6794252 & 129025 & 40069 & 130 & 6.33 & 2.72 \\
\hline
\end{tabular}

Table 13: 2D Driven cavity - increasing Reynolds-number, grid-size $n_{x}=512$

\begin{tabular}{|c|r|r|r|r|r|r|r|}
\hline$n_{x}$ & $\mathrm{~N}$ & $\mathrm{nnz}$ & $\mathrm{N}_{\mathrm{S}}$ & $\mathrm{n}$ & iter & fill 1 & fill 2 \\
\hline 64 & 12160 & 103820 & 1793 & 533 & 185 & 6.09 & 0.418 \\
128 & 48896 & 420620 & 7681 & 2341 & 181 & 6.22 & 0.953 \\
256 & 196096 & 1693196 & 31745 & 9797 & 167 & 6.29 & 1.75 \\
512 & 785408 & 6794252 & 129025 & 40069 & 130 & 6.33 & 2.72 \\
\hline
\end{tabular}

Table 14: 2D Driven cavity - grid refinement at $R e=8000$

\section{Discussion and conclusions}

In this paper we have shown that the structure preserving complete $L D L^{T}$ factorization introduced in De Niet \& Wubs (2009) of an $\mathcal{F}$-matrix can be transformed into an incomplete factorization. We constructed an iterative solver for the whole system, which avoids having to balance inner and outer iterations as in a segregated approach. Depending only on a single parameter (the subdomain size), the method is as easy to use as a direct solver and gives reliable results in a reasonable turn-around time.

For Stokes matrices we were able to prove grid-independent convergence. The total number of operations required is currently not grid-independent since we use a direct solver to solve the reduced system. However, the amount of work required for this step is reduced by about the cube of the subdomain size in $2 \mathrm{D}$ and the sixth power in 3D. So increasing the subdomain size by a factor 2 means in $2 \mathrm{D}$ a factor 8 and in $3 \mathrm{D}$ a factor 64 . For the Navier-Stokes equations we also observed grid-independent convergence. We are developing a parallel $\mathrm{C}++$ implementation of the method that can be applied recursively, making it a multi-level method.

We proved the robustness of the method for Stokes and Navier-Stokes equations, where in the latter case the matrix should be definite. Computations show that the method still performs well for cases where eigenvalues pass the imaginary axis away from the origin (Hopf bifurcations).

In the case of $\mathcal{F}$-matrices we are able to keep the computation in the kernel of the constraint equation, i.e. for Stokes in the divergence free space, allowing us to use the CG method. Though the $\mathcal{F}$-matrices seem to be a limited class due to the constraints on the sparsity pattern in $B$, many applications lead to matrices of this type. 


\section{Acknowledgements}

Part of this work was done during a sabbatical leave of the first author to the Numerical Analysis Group at Rutherford Appleton Laboratory in Chilton (UK). We kindly thank that group for the hospitality and good-fellowship. The research of the second author was funded by the Netherlands Organization for Scientific Research, NWO, through the contract ALW854.00.028.

\section{References}

Amestoy, P.R., Davis, T.A. And Duff, I.S. (1996) An approximate minimum degree ordering algorithm, SIAM J. Matrix Anal. Appl., 17(4), 886905 .

Amestoy, P.R., Davis, T.A. And Duff, I.S. (2004) Algorithm 837: AMD, an approximate minimum degree ordering algorithm, ACM Trans. Math. Software, 30(3):381-388.

Arioli, M. And Manzini, G. (2003) Null space algorithm and spanning trees in solving Darcy's equation, BIT, 43(5), 839-848.

Axelsson, O. AND LARIN, M (1997) An algebraic multilevel iteration method for finite element matrices, J. Comp. Appl. Math., 89:135-153.

Axelsson, O. (1994) Iterative Solution Methods, Cambridge University Press.

Benzi, M., Golub, G.H. And Liesen, J. (2005) Numerical solution of saddle point problems, Acta Numer., 14:1-137.

Benzi, M. And OlshanskiI, M.A. (2006) An Augmented Lagrangian-Based Approach to the Oseen Problem, SIAM J. Scientific Computing, 28:20952113.

Botta, E.F.F. And Wubs, F.W. (1999) Matrix Renumbering ILU: An effective algebraic multilevel ILU-preconditioner for sparse matrices, SIAM J. Matrix Anal. Appl., 20(4):1007-1026.

Eiskhout, V. (1992) Beware of unperturbed modified incomplete factorizations, Iterative Methods in Linear Algebra, edited by, R. Beauwens and P. de Groen, p. 583, Elsevier Science, North-Holland, 1992.

Duff, I.S., Erisman, A.M. And Reid, J.K. (1986) Direct methods for sparse matrices, Monographs on Numerical Analysis, Oxford University Press.

Elman, H.C., Silvester, D.J. And Wathen, A.J. (2002) Performance and analysis of saddle point preconditioners for the discrete steady-state NavierStokes equations, Numer. Math., 90(4):665-688. 
Elman, H., Howle, V.E., Shadid, J., Shuttleworth, R., and TumiNARO, R. A taxonomy and comparison of parallel block multi-level preconditioners for the incompressible Navier-Stokes equations, J. Comput. Phys., 227:1790-1808, 2008

Gaidamour, J. And HÉnOn, P. (2008) A parallel direct/iterative solver based on a Schur complement approach, Proceedings of IEEE 11th International Conference on Computational Science and Engineering, Sao Paulo, Brazil, p.98-105.

George, A. (1973) Nested dissection of a regular finite element mesh, SIAM J. Numer. Anal., 10:345-363.

George, A. And Liu, J.W.H. (1981) Computer solution of large sparse positive definite systems, Prentice-Hall Series in Computational Mathematics, Prentice-Hall.

Gould, N. I. M., Hribar, M.E. And Nocedal J. (2001) On the solution of equality constrained quadratic programming problems arising in optimization, SIAM J. Sci. Comput., 23:13761395.

HÉnon, P. And SAAD, Y. (2006) A Parallel Multistage ILU Factorization Based on a Hierarchical Graph Decomposition. SIAM J. of Sci. Comput., 28:2266-2293

KaryPis, G. AND Kumar, V. (1998) A fast and high quality multilevel scheme for partitioning irregular graphs, SIAM J. Sci. Comput., 20(1):359-392.

Kay, D., Loghin, D. \& Wathen, A.J. (2002) A preconditioner for the steadystate Navier-Stokes equations, SIAM J. Sci. Comput., 24(1):237-256.

Keller, C., Gould, N. I. M. And Wathen, A. J. (2000) Constraint Preconditioning for Indefinite Linear Systems, SIAM J. Matrix Anal. Appl, 21:1300-1317.

Lust, K., Roose, D., Spence, A. And Champneys, A.R.(1999) An adaptive Newton-Picard algorithm with subspace iteration for computing periodic solutions SIAM J. Sci. Comput., 19(4):1188-1209, 1998.

Meurant, G. (1999) Computer solution of large linear systems, North-Holland.

MeiJerink, J.A. AND VAn DER Vorst, H.A. (1977) An iterative solution method for linear systems of which the coefficient matrix is a symmetric M-matrix, Math. Comp.131:148-162.

Niet, A.C.DE AND Wubs, F.W. (2007) Two saddle point preconditioners for fluid flows, Int. J. Numer. Methods Fluids, 54(4):355-377.

Niet, A.C.DE AND Wubs, F.W. (2009) Numerically stable $L D L^{T_{-}}$ factorization of F-type saddle point matrices, IMA J. Numer. Anal., 29:208-234, 2009. 
NotAy, Y (2010) An aggregation-based algebraic multigrid method, ETNA,To appear

Pellegrini, F. SCOTCH 5.1 User's guide (2008), Technical report, LaBRI.

Sleijpen, G.L.G. And Wubs, F.W.(2003) Exploiting Multilevel Preconditioning Techniques in Eigenvalue Computations, SIAM J. Sci. Comput., 25(4):1249-1272.

Tiesinga, G., Wubs, F.W. And Veldman, A.E.P. (2002) Bifurcation analysis of incompressible flow in a driven cavity by the Newton-Picard method, J. Comput. Appl. Math.

Toselli, A. And Widlund, O. (2005) Domain decomposition methods - Algorithms and theory, Springer.

TŮmA, M. (2002) A note on the $L D L^{T}$ decomposition of matrices from saddlepoint problems, SIAM J. Matrix Anal. Appl., 23(4):903-915.

VAVASIS, S.A. (1994) Stable numerical algorithms for equilibrium systems, SIAM J. Matrix Anal. Appl., 15(4):1108-1131.

VORst, H.A. VAN DER (2003) Iterative Krylov methods for large linear systems, volume 13 of Cambridge Monographs on Applied and Computational Mathematics, Cambridge University Press.

Verstappen, R.C.W.P. And Veldman, A.E.P. (2003) Symmetry-preserving discretisation of turbulent flow, J. Comp. Phys., 187:343-368.

Wubs, F.W., De Niet, A.C. And H.A. Dijkstra, The Performance of Implicit Ocean models on B- and C-grids, J. Comput. Phys., 211:210-228. 